\title{
Surface Coating Effect on Si Nanowires Anodes for Lithium Ion Batteries
}

\author{
Langli Luo ${ }^{1}$, Pengfei Yan ${ }^{1}$, Ji-Guang Zhang ${ }^{2}$, Chunmei Ban ${ }^{3}$ and Chongmin Wang ${ }^{1}$ \\ ${ }^{1}$ Environmental Molecular Sciences Laboratory, Pacific Northwest National Laboratory, 902 Battelle \\ Boulevard, Richland, WA 99352, USA \\ ${ }^{2}$ Energy and Environmental Directorate, Pacific Northwest National Laboratory, 902 Battelle Boulevard, \\ Richland, WA 99352, USA \\ ${ }^{3}$ National Renewable Energy Laboratory, 1617 Cole Boulevard, Golden, CO, 80401, USA
}

Silicon based materials holds promise for next generation negative electrode for high-capacity Li-ion batteries, yet tremendous research effort have been made for tackling the chemo-mechanical failure that is associated with the intrinsic large volume change of Si during lithiation and delithiation process. Surface modification of Si nanostructures have successfully alleviated this problem and improved the cyclability of Si based anodes. Successful surface modifications are expected to provide both good protection and conduction between Si nanostructures without sacrificing the electrochemical performance. However, how these surface modifications will affect the lithiation and delithiation behavior of the Si nanostructures and whey they improve or deteriorate the performance of electrodes are largely unknown.

The emerging in-situ transmission electron microscopy (TEM) techniques with localized electrical measurement capabilities provide a practical platform for investigating electrochemical reactions in Liion battery materials by building a full or half "nano-cell" inside the TEM specimen chamber [2]. Such real-time observations of dynamic composition and microstructural evolution in the electrochemical reaction have provided many novel clues to understand the lithiation/de-lithiation mechanisms at nano or atomic-scale for several novel anode materials. Herein, we report a comparative study of surface coatings effect on the lithiation and delithiation behavior and kinetics of Si nanowires and nanoparticles.

Fig. 1 $\mathrm{A}$ and $\mathrm{B}$ show time-resolved TEM images in top and bottom panel depict the typical lithiation process for alucone and $\mathrm{Al}_{2} \mathrm{O}_{3}$ coated $\mathrm{Si}$ nanowires with same diameter, respectively. The alucone coated Si nanowire has a clear $\mathrm{V}$ shaped lithiation profile comparing with symmetrical linear lithiation file of $\mathrm{Al}_{2} \mathrm{O}_{3}$ coated $\mathrm{Si}$ nanowire as shown in the corresponding schematic drawings. $\mathrm{Al}_{2} \mathrm{O}_{3}$ coating with a dense structure but poor Li ion conductivity leads to a symmetrical lithiation profile along the axial direction of the Si nanowires and a slower lithiation rate, while alucone coating with a less dense structure and fast $\mathrm{Li}$ ion transport yields to a typical V shape lithiation profile along the axial direction of the Si nanowire and high rate lithiation. The lithiation depth vs. time for two type of Si nanowires is illustrated in Fig. 1C that a much faster initial lithiation rate is found for alucone coated Si nanowire.

Fig. 2 illustrates the risk for pulverization of Si nanostructures or damage to functional surface coatings through vigorous alloying reaction. Upon biasing, the no. 4 nanowire lithiated with two distinct manners on upper and lower part of the nanowire. The upper part connected to no. 2 nanowire with a larger diameter alloyed aggressively to form amorphous $\mathrm{Li}_{x} \mathrm{Si}$ causing pulverization of half of the nanowire and $\mathrm{Al}_{2} \mathrm{O}_{3}$ coating is also destroyed. On the contrary, the lower part of the nanowire connected to no. 3 with a smaller diameter Si was lithiated smoothly and the surface coating was preserved during lithiation. This variation 
in lithiation behavior in one $\mathrm{Si}$ nanowire is largely attributed to the diffusion paths of $\mathrm{Li}$ ions. Once the reaction barrier is reached, large chemical potential drives the alloying reaction fast enough to induce large volume expansion that cannot be accommodated by the materials system leading to pulverization.

Reference:

[1] Arico, A. S., Bruce, P., Scrosati, B., Tarascon, J.-M. \& van Schalkwijk, W. Nat Mater 4 (2005), 366.

[2] Huang, J. Y. Zhong, L., Wang, C. M. et al. Science 330 (2010), 1515.
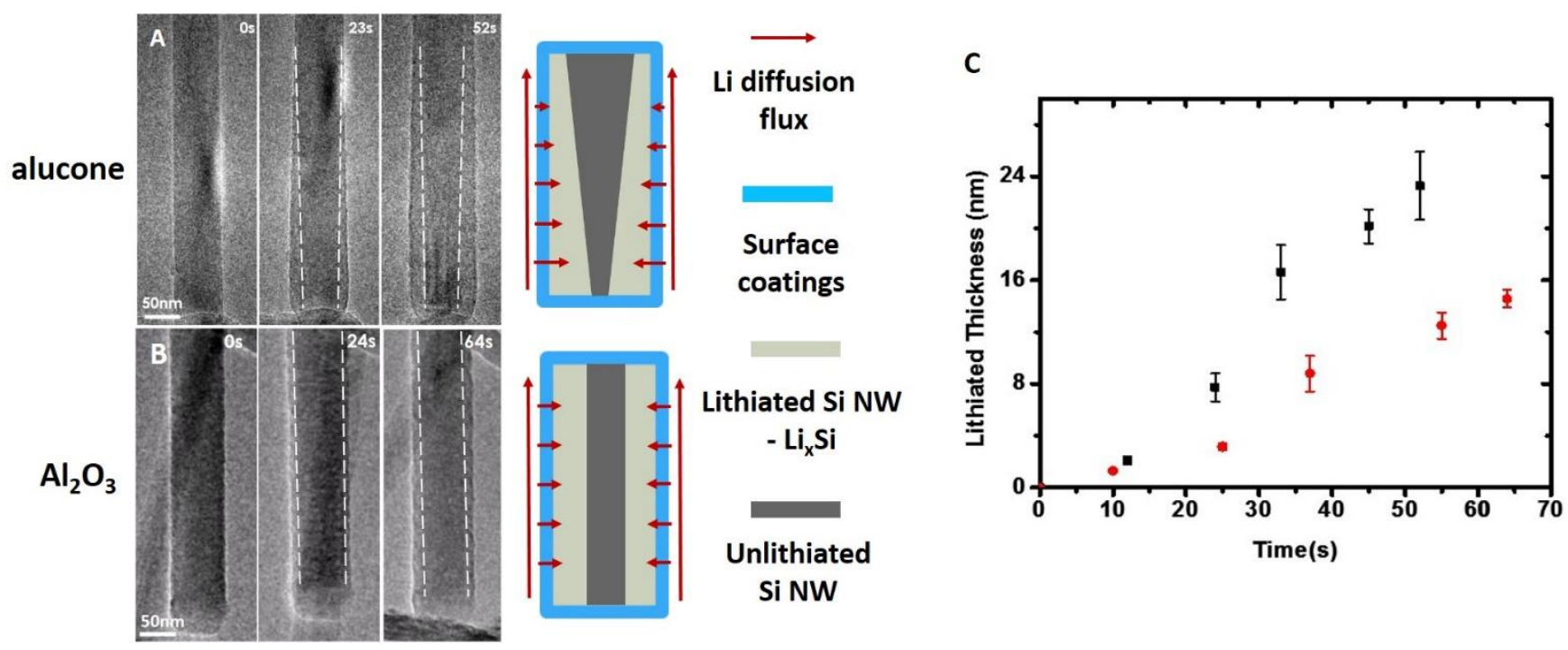

Figure $1 \mathrm{~A}$ ) and B) Time-resolved TEM images show the development of lithiation profiles of alucone and $\mathrm{Al}_{2} \mathrm{O}_{3}$ coated Si nanowires and schematic Li diffusion paths through the Si NWs which determines the lithiation behavior and kinetics; C) Average thickness of lithiated vs. time for alucone (black square) and $\mathrm{Al}_{2} \mathrm{O}_{3}$ (red dot) coated $\mathrm{Si}$ nanowires.
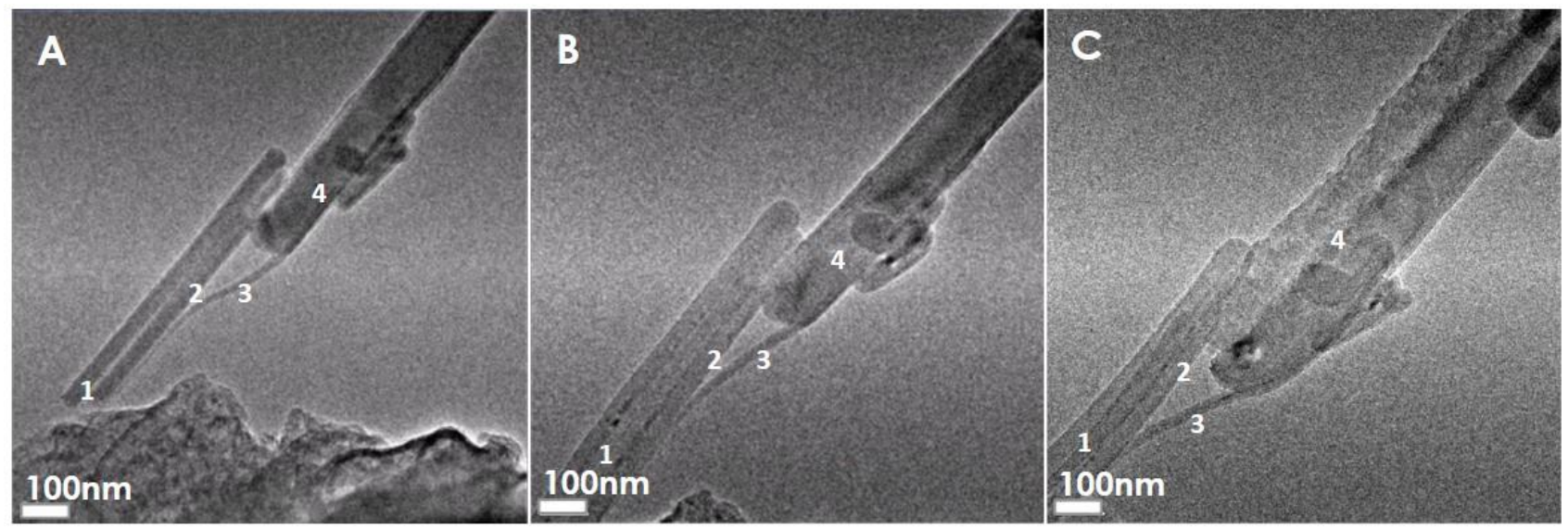

Figure 2. (A-C) Time sequential TEM images of failure of $\mathrm{Al}_{2} \mathrm{O}_{3}$ coating on Si nanowire during lithiation process. 